\title{
The Alpha Power Transformed Dagum Distribution: Properties and Applications
}

\author{
Hesham Reyad', Farrukh Jamal', G.G. Hamedani ${ }^{3, *}$ and Soha Othman ${ }^{4}$ \\ 1 Department of Information Systems and Production Management, \\ College of Business and Economics, Qassim University, KSA \\ hesham_reyad@yahoo.com \\ 2Department of Statistics, Govt.S.A. Postgraduate College, Dera Nawab Sahib, Pakistan \\ Drfarrukh1982@gmail.com \\ ${ }^{3}$ Department of Mathematics, Statistics and Computer Science, Marquette University, USA \\ g.hamedani@mu.edu \\ ${ }^{4}$ Department of Applied Statistics, Cairo University, Egypt \\ soha_othman@yahoo.com \\ "Correspondence: g.hamedani@mu.edu
}

\begin{abstract}
In this study, we propose a new extension of the Dagum distribution called the alpha power transformed Dagum distribution. Basic statistical properties of the new distribution such as; quantile function, raw and incomplete moments, moment generating function, order statistics, Rényi entropy, stochastic ordering and stress strength model are investigated. The characterizations of the new model is investigated. The method of maximum likelihood is used to estimate the model parameters of the new distribution and the observed information matrix is also obtained. A Monte Carlo simulation is presented to examine the behavior of the parameter estimates. The applicability of the new model is demonstrated by means of three applications.
\end{abstract}

\section{Introduction}

The Dagum distribution was introduced by Dagum (1977) for analysing income data as an alternative model to the log-normal and Pareto distributions. This distribution has been used in different fields such as, reliability and survival analysis,

Received: 24 Sep 2021.

Key words and phrases. alpha-power transformed family; characterizations; Dagum distribution; probability weighted moments; Rényi entropy; stochastic ordering; stress strength model. 
wealth and income data and meteorological data. The Dagum distribution has cumulative distribution function (cdf) given by

$$
F(x)=\left(1+\lambda x^{-\beta}\right)^{-\theta}, x \geq 0
$$

and its corresponding probability density function (pdf) is

$$
f(x)=\lambda \beta \theta x^{-\beta-1}\left(1+\lambda x^{-\beta}\right)^{-\theta-1}, x>0,
$$

where $\lambda>0$ is the scale parameter and $\beta, \theta>0$ are the shape parameters.

There are many extensions of the Dagum distribution such as; Beta-Dagum by Domma and Condino (2013), weighted Dagum by Oluyede and Ye (2014), Weibull Dagum by Tahir et al. (2016), power log Logistic Dagum by Bakouch et al. (2017) and odd log Logistic Dagum by Domma et al. (2018).

Moreover, if $g(x ; \phi)$ and $G(x ; \phi)$ denote the pdf and cdf of a baseline model with parameter vector $\phi$. Mahdavi and Kundu (2017) introduced a new method of generating continuous distributions called the alpha-power transformation (APT for short) with cdf and pdf given by

$$
\begin{aligned}
& F(x ; \phi)=\frac{\alpha^{G(x ; \phi)}-1}{\alpha-1}, \alpha>0, \alpha \neq 1, x \in R, \\
& f(x ; \phi)=\frac{\log (\alpha)}{\alpha-1} g(x ; \phi) \alpha^{G(x ; \phi)}, \alpha>0, \alpha \neq 1, x \in R .
\end{aligned}
$$

The goal of this article is to derive a new distribution from the Dagum distribution by Alpha-power transformation as suggested by Mahdavi and Kundu (2017), called alpha power transformed Lindley (APTL) distribution. This concept of generalization is well established in the statistical literature (see Dey et al. (2017a, 2017b)). The proposed distribution encompasses the behavior of and provides better fits than some well known lifetime distributions in the literature; please see in application section 6. We are motivated to introduce the alpha power transformed Dagum (APTD for short) distribution because (i) it is capable of modeling increasing, decreasing, constant, bathtub and upside-down bathtub shaped hazard rates; (ii) it can be viewed as a suitable model for fitting the skewed data which may not be properly fitted by other known distributions and can also be used in a variety of problems in different 
areas; see also in application section 6; and (iii) three real data applications show that it compares well with other competing lifetime distributions. The cdf and pdf of the APTD distribution are given, respectively, by

$$
\begin{aligned}
& F(x ; \phi)=\frac{\alpha^{\left(1+\lambda x^{-\beta}\right)^{-\theta}}-1}{\alpha-1}, \alpha>0, \alpha \neq 1, \lambda, \beta, \theta>0, x \geq 0, \\
& f(x ; \phi)=\frac{\lambda \beta \theta \log (\alpha)}{\alpha-1} x^{-\beta-1}\left(1+\lambda x^{-\beta}\right)^{-\theta-1} \alpha^{\left(1+\lambda x^{-\beta}\right)^{-\theta}}, x>0 .
\end{aligned}
$$

Henceforth, a random variable with density (6) will be denoted by $X \sim \operatorname{APTD}(\alpha, \beta, \lambda, \theta)$. The hazard function $\tau(x)$ for the APTD distribution is given by

$$
\tau(x)=\frac{\lambda \beta \theta \log (\alpha) x^{-\beta-1}\left(1+\lambda x^{-\beta}\right)^{-\theta-1} \alpha^{\left(1+\lambda x^{-\beta}\right)^{-\theta}}}{\alpha^{\left(1+\lambda x^{-\beta}\right)^{-\theta}}-1}, x>0 .
$$

The remainder of this paper is orgainzed as follows. In Section 2, main mathematical properties of the APTD model are studied. In Section 3, certain characterizations of the new disatribution are presented. In Section 4, the maximum likelihood estimates are obtained for the model parameters. A simulation study is conducted in Section 5. In Section 6, we provide three applications. Section 7 offers some concluding remarks.

\section{Mathematical Properties}

In this section, we will study some main properties of the APTD distribution.

\subsection{Quantile Function}

The quantile function of the APTD distribution $Q(u)=F^{-1}(u)$ for $u \in(0,1), \alpha>0$, $\beta>0, \lambda>0$ and $\theta>0$ is the solution of the non-linear equation

$$
Q(u)=G^{-1}\left\{\left(\frac{1}{\lambda}\left[\left\{\frac{\log (1+(\alpha-1) u)}{\log (\alpha)}\right\}^{-1 / \theta}-1\right]\right)^{-1 / \beta}\right\} .
$$

\subsection{The Shape of the APTD Distribution}

The shape of the density and hazard functions of the APTD distribution can be described mathematically. The critical points of the density function are the roots of the following equation: 


$$
-\left(\frac{\beta+1}{x}\right)+\left(\frac{\lambda \beta(\theta+1) x^{-\beta-1}}{1+\lambda x^{-\beta}}\right)+\left\{\frac{\lambda \beta \theta \log (\alpha) x^{-\beta-1}}{\left(1+\lambda x^{-\beta}\right)^{\theta+1}}\right\}=0 .
$$

Further, the critical points of the hazard function are the roots of the following equation:

$$
\begin{aligned}
& -\left(\frac{\beta+1}{x}\right)+\left(\frac{\lambda \beta(\theta+1) x^{-\beta-1}}{1+\lambda x^{-\beta}}\right)+\left\{\frac{\lambda \beta \theta \log (\alpha) x^{-\beta-1}}{\left(1+\lambda x^{-\beta}\right)^{\theta+1}}\right\} \\
& -\left\{\frac{\lambda \beta \theta \log (\alpha) x^{-\beta-1}\left(1+\lambda x^{-\beta}\right)^{-\theta-1} \alpha^{\left(1+\lambda x^{-\beta}\right)^{-\theta}}}{\alpha^{\left(1+\lambda x^{-\beta}\right)^{-\theta}}-1}\right\}=0 .
\end{aligned}
$$

Some plots of the density and hazard functions are displayed in Figures 1 and 2.
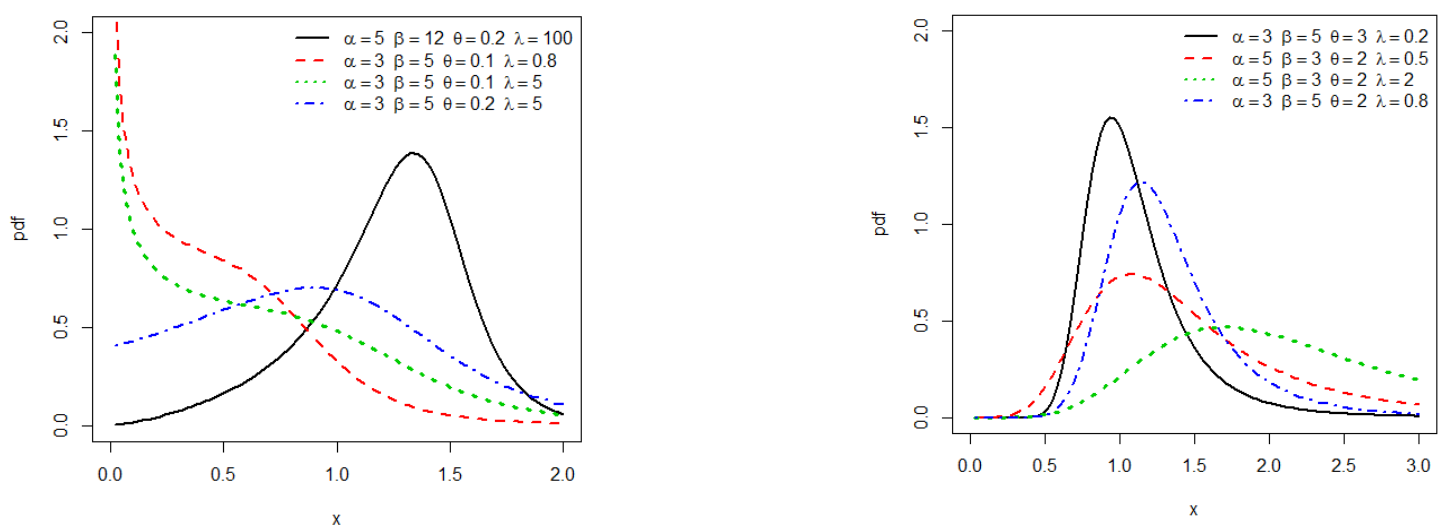

Figure 1: Plots of the APTD pdf for selected parameter values.
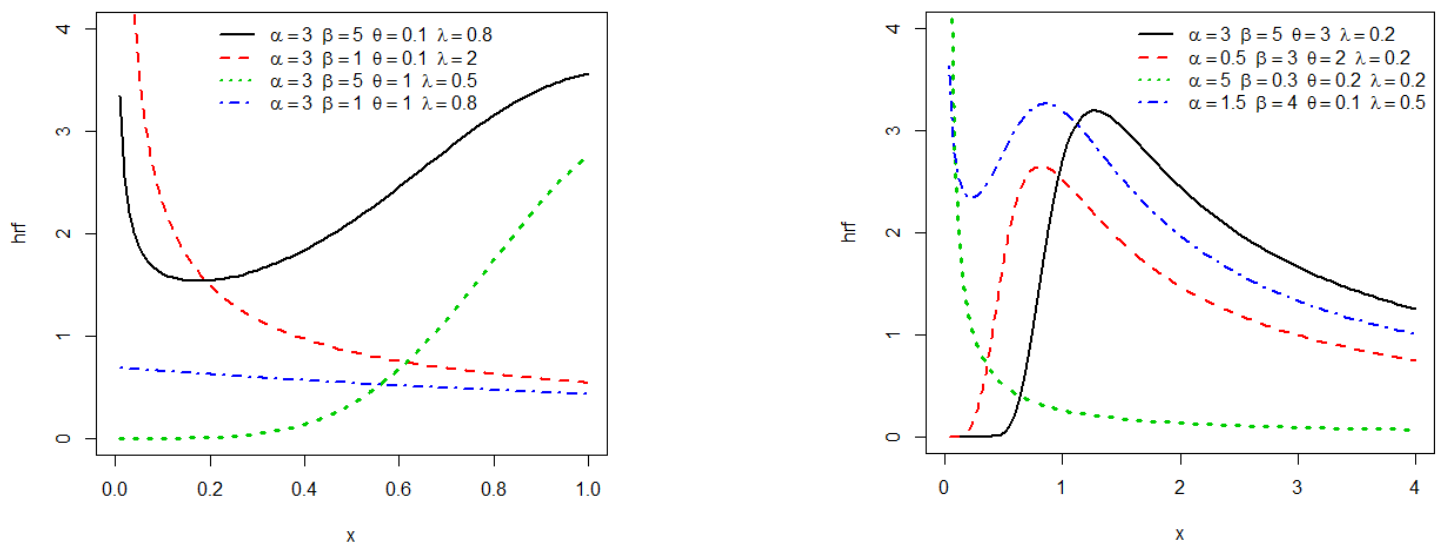

Figure 2: Plots of the APTD hrf for selected parameter values. 


\subsection{Moments and Moment Generating Function}

Let $X$ be a random variable with the APTD distribution, then the ordinary moment, say $\mu_{r}^{\prime}$, is given by

$$
\begin{aligned}
\mu_{r}^{\prime} & =E\left(X^{r}\right)=\int_{-\infty}^{\infty} x^{r} f(x) d x \\
& =\sum_{w=0}^{\infty} \frac{\lambda \beta \theta(\log \alpha)^{w+1}}{(\alpha-1) w !} \int_{0}^{\infty} x^{r-\beta-1}\left(1+\lambda x^{-\beta}\right)^{-\theta(w+1)-1} d x \\
& =\sum_{w=0}^{\infty} \frac{\lambda^{-\theta(w+1)} \beta \theta(\log \alpha)^{w+1}}{(\alpha-1) w !} \int_{0}^{\infty} x^{r+\beta \theta(w+1)-1}\left(1+\frac{x^{\beta}}{\lambda}\right)^{-\theta(w+1)-1} d x .
\end{aligned}
$$

Letting $y=\left(1+x^{\beta} / \lambda\right)^{-1}$, we obtain

$$
\begin{aligned}
\mu_{r}^{\prime} & =\sum_{w=0}^{\infty} \frac{\lambda^{r / \beta} \theta(\log \alpha)^{w+1}}{(\alpha-1) w !} \int_{0}^{1} y^{-r / \beta}(1-y)^{r / \beta+\theta(w+1)-1} d y \\
& =\sum_{w=0}^{\infty} \frac{\lambda^{r / \beta} \theta(\log \alpha)^{w+1}}{(\alpha-1) w !} B(1-r / \beta, r / \beta+\theta(w+1)),
\end{aligned}
$$

where, $B(m, n)=\int_{0}^{1} x^{m-1}(1-x)^{n-1} d x$ is the beta function. Substituting $r=1,2,3,4$ in (9), we obtain the mean $=\mu_{1}^{\prime}$, variance $=\mu_{2}^{\prime}-\mu_{1}^{\prime 2}$, skewness $=\mu_{3}^{\prime} / \mu_{1}^{\prime 3 / 2}$ and kurtosis $=\mu_{4}^{\prime} / \mu_{1}^{\prime 2}$.

The plots of the skewness and kurtosis of the APTD distribution for parameters $\alpha=0.5, \beta=0.8$ are displayed in Figure (3). Based on these plots, we conclude: if the parameters $\alpha$ and $\beta$ increase, the skewness decreases and kurtosis increases. Moreover, parameter $\beta$ has more significant effect on skewness and kurtosis measures than parameter $\alpha$.
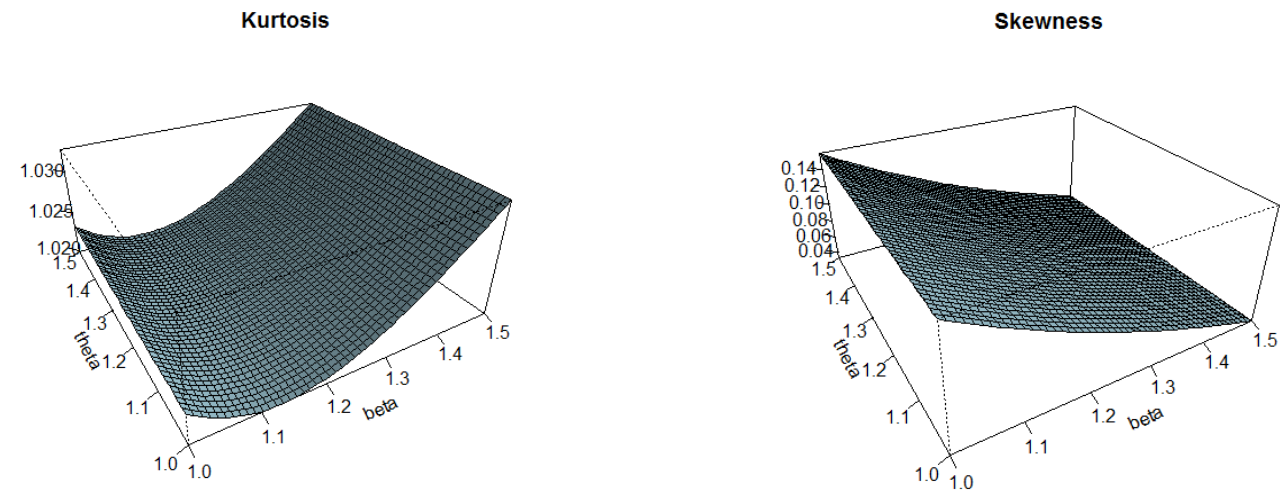

Figure 3: The skewness and kurtosis of the APTD distribution. 
The nth central moment of the APTD distribution, say $\mu_{n}$, can be obtained from

$$
\begin{aligned}
\mu_{n} & =\sum_{r=0}^{n}\left(\begin{array}{l}
n \\
r
\end{array}\right)\left(-\mu_{1}^{\prime}\right)^{n-r} E\left(x^{r}\right) \\
& =\sum_{r=0}^{n} \sum_{w=0}^{\infty}\left(\begin{array}{l}
n \\
r
\end{array}\right) \frac{\left(-\mu_{1}^{\prime}\right)^{n-r} \lambda^{r / \beta} \theta(\log \alpha)^{w+1}}{(\alpha-1) w !} B(1-r / \beta, r / \beta+\theta(w+1)) .
\end{aligned}
$$

The $r$ th incomplete moment of the APTD distribution, denoted by $\varphi_{s}(t)$, is

$$
\begin{aligned}
\varphi_{s}(t) & =\int_{-\infty}^{t} x^{s} f(x) d x \\
& =\sum_{w=0}^{\infty} \frac{\lambda \beta \theta(\log \alpha)^{w+1}}{(\alpha-1) w !} \int_{0}^{t} x^{s-\beta-1}\left(1+\lambda x^{-\beta}\right)^{-\theta(w+1)-1} d x \\
& =\sum_{w, j=0}^{\infty} \frac{\lambda^{j+1} \beta \theta(\log \alpha)^{w+1}}{(\alpha-1) w !}\left(\begin{array}{c}
-\theta(w+1)-1 \\
j
\end{array}\right) \int_{0}^{t} x^{s-\beta(j+1)-1} d x \\
& =\sum_{w, j=0}^{\infty}\left(\begin{array}{c}
-\theta(w+1) \\
j
\end{array}\right) \frac{\lambda^{j+1} \beta \theta(\log \alpha)^{w+1} t^{s-\beta(j+1)}}{(\alpha-1)(s-\beta(j+1)) w !} .
\end{aligned}
$$

The moment generating function of the APTD distribution, denoted by $M_{X}(t)$, can be obtained by

$$
\begin{aligned}
M_{X}(t)= & E\left(e^{t X}\right)=\sum_{r=0}^{\infty} \frac{t^{r}}{r !} E\left(X^{r}\right) \\
& =\sum_{r, w=0}^{\infty} \frac{t^{r} \lambda^{r / \beta} \theta(\log \alpha)^{w+1}}{(\alpha-1) w ! r !} B\left(1-\frac{r}{\beta}, \frac{r}{\beta}+\theta(w+1)\right) .
\end{aligned}
$$

\subsection{Probability Weighted Moments}

The $(r+s)$ th PWM of a random variable $X$ with the APTD distribution, say $M_{r, s}$, is given by

$$
M_{r, s}=E\left(X^{r} F(x)^{s}\right)=\int_{-\infty}^{\infty} X^{r} F(x)^{s} f(x) d x .
$$

From (5) and (6), we have 


$$
\begin{gathered}
f(x) F(x)^{s}=\frac{\lambda \beta \theta \log (\alpha)}{\alpha-1} x^{-\beta-1}\left(1+\lambda x^{-\beta}\right)^{-\theta-1} \alpha^{\left(1+\lambda x^{-\beta}\right)^{-\theta}}\left\{\frac{\alpha^{\left(1+\lambda x^{-\beta}\right)^{-\theta}}-1}{\alpha-1}\right\} \\
=\sum_{j=0}^{\infty} \frac{(-1)^{j} \lambda \beta \theta \log (\alpha)}{(\alpha-1)^{s+1}}\left(\begin{array}{c}
s \\
j
\end{array}\right)^{-\beta-1}\left(1+\lambda x^{-\beta}\right)^{-\theta-1} \alpha^{(s-j+1)\left(1+\lambda x^{-\beta}\right)^{-\theta}} \\
=\sum_{j, w=0}^{\infty} \frac{(-1)^{j} \lambda \beta \theta(\log (\alpha))^{w+1}(s-j+1)^{w}}{(\alpha-1)^{s+1} w !}\left(\begin{array}{c}
s \\
j
\end{array}\right) \\
\times x^{-\beta-1}\left(1+\lambda x^{-\beta}\right)^{-\theta(w+1)-1} .
\end{gathered}
$$

Subsituting from (14) in (13), we obtain

$$
\begin{array}{r}
M_{r, s}=\sum_{j, w=0}^{\infty} \frac{(-1)^{j} \lambda^{r / \beta} \theta(\log (\alpha))^{w+1}(s-j+1)^{w}}{(\alpha-1)^{s+1} w !}\left(\begin{array}{c}
s \\
j
\end{array}\right) \\
\times B(1-r / \beta, r / \beta+\theta(w+1)) .
\end{array}
$$

\subsection{Order Statistics}

Let $X_{1: n} \leq X_{2: n}, \ldots \leq X_{n: n}$ be order statistics corresponding to a sample of size $n$ from the APTD distribution. The pdf of $X_{k: n}$, the $k$ th order statistic, is given by

$$
f_{X_{k: n}}(x)=\frac{1}{B(k, n-k+1)} \sum_{i=0}^{n-k}(-1)^{i}\left(\begin{array}{c}
n-k \\
i
\end{array}\right) f(x) F(x)^{k+i-1} .
$$

Based on (5) and (6), we have

$$
\begin{aligned}
f(x) F(x)^{k+i-1}=\sum_{j=0}^{\infty} \frac{(-1)^{j} \lambda \beta \theta \log (\alpha)}{(\alpha-1)^{k+i}}\left(\begin{array}{c}
k+i-1 \\
j
\end{array}\right) \\
\quad \times x^{-\beta-1}\left(1+\lambda x^{-\beta}\right)^{-\theta-1} \alpha^{(k+i-j)\left(1+\lambda x^{-\beta}\right)^{-\theta}} .
\end{aligned}
$$

Using (17) in (16), we have

$$
\begin{array}{r}
f_{X_{k \cdot n}}(x)=\sum_{i=0}^{n-k} \sum_{j=0}^{\infty} \frac{(-1)^{j+j} \lambda \beta \theta \log (\alpha)}{(\alpha-1)^{k+i} B(k, n-k+1)}\left(\begin{array}{c}
n-k \\
i
\end{array}\right)\left(\begin{array}{c}
k+i-1 \\
j
\end{array}\right) \\
\times x^{-\beta-1}\left(1+\lambda x^{-\beta}\right)^{-\theta-1} \alpha^{(k+i-j)\left(1+\lambda x^{-\beta}\right)^{-\theta}} .
\end{array}
$$

Furthermore, the $r$ th moment of $k$ th order statistic for the APTD distribution is given by 


$$
\begin{gathered}
E\left(x_{k: n}^{r}\right)=\sum_{i=0}^{n-k} \sum_{j=0}^{\infty} \frac{(-1)^{j+j} \lambda \beta \theta \log (\alpha)}{(\alpha-1)^{k+i} B(k, n-k+1)}\left(\begin{array}{c}
n-k \\
i
\end{array}\right)\left(\begin{array}{c}
k+i-1 \\
j
\end{array}\right) \\
\quad \times \int_{0}^{\infty} x^{r-\beta-1}\left(1+\lambda x^{-\beta}\right)^{-\theta-1} \alpha^{(k+i-j)\left(1+\lambda x^{-\beta}\right)^{-\theta}} d x \\
=\sum_{i=0}^{n-k} \sum_{j, w=0}^{\infty} \frac{(-1)^{j+j} \lambda \beta \theta(\log (\alpha))^{w+1}(k+i-j)^{w}}{(\alpha-1)^{k+i} w ! B(k, n-k+1)}\left(\begin{array}{c}
n-k+1 \\
i
\end{array}\right)\left(\begin{array}{c}
k+1 \\
j
\end{array}\right) \\
\times \int_{0}^{\infty} x^{r-\beta-1}\left(1+\lambda x^{-\beta}\right)^{-\theta(w+1)-1} d x \\
E\left(x_{k: n}^{r}\right)=\sum_{i=0}^{n-k} \sum_{j, w=0}^{\infty} \frac{(-1)^{j+j} \lambda^{r / \beta} \theta(\log (\alpha))^{w+1}(k+i-j)^{w}}{(\alpha-1)^{k+i} w !}\left(\begin{array}{c}
n-k \\
i
\end{array}\right)\left(\begin{array}{c}
k+i-1 \\
j
\end{array}\right) \\
\times \frac{B(1-r / \beta, r / \beta+\theta(w+1))}{B(k, n-k+1} .
\end{gathered}
$$

\subsection{Rényi Entropy}

The entropy is a useful approach used in different areas such as queuing theory and statistics.The Rényi entropy is defined as

$$
I_{R}(X)=(1-\mu)^{-1} \log \int_{-\infty}^{\infty} f(x)^{\mu} d x, \mu>0, \mu \neq 0 .
$$

Using (6) and after some manipulations, we have

$$
I_{R}(X)=(1-\mu)^{-1} \log \left\{\begin{array}{l}
\sum_{w=0}^{\infty} \frac{\beta^{\mu-1} \theta^{\mu}(\log (\alpha))^{\mu+w} \mu^{w}}{w ! \lambda^{(\mu-1) / \beta}} \\
\times B(\mu+(\mu-1) / \beta, \theta(\mu+w)-(\mu-1) / \beta)
\end{array}\right\} .
$$

\subsection{Stochastic Ordering}

Stochastic ordering is a vital criterion that is used in different fields to examine the comparative behavior. According to Shaked and Shanthikumar (2007), a random variable $X_{1}$ is said to be smaller than another random variable $X_{2}$ in the likelihood ratio order $\left(X_{1} \leq_{l r} X_{2}\right)$ if $f_{1}(x) / f_{2}(x)$ decreases in $x$. The following theorem shows that the APTD distribution is ordered in likelihood ratio ordering if the appropriate assumptions exist. 
Theorem 1: Let $X_{1} \sim \operatorname{APTD}\left(\alpha_{1}, \beta_{1}, \lambda_{1}, \theta_{1}\right)$ and $X_{2} \sim \operatorname{APTD}\left(\alpha_{2}, \beta_{2}, \lambda_{2}, \theta_{2}\right)$. If $\alpha_{1}=\alpha_{2}$, $\lambda_{1}=\lambda_{2}, \theta_{1}=\theta_{2}$ and $\beta_{1} \geq \beta_{2}$, then $X_{1} \leq_{l r} X_{2}$.

Proof: We have

$$
\frac{f_{1}(x)}{f_{2}(x)}=\frac{\frac{\lambda_{1} \beta_{1} \theta_{1} \log \left(\alpha_{1}\right)}{\alpha_{1}-1} x^{-\beta_{1}-1}\left(1+\lambda_{1} x^{-\beta_{1}}\right)^{-\theta_{1}-1} \alpha_{1}^{\left(1+\lambda_{1} x^{-\beta_{1}}\right)^{-\theta_{1}-1}}}{\frac{\lambda_{2} \beta_{2} \theta_{2} \log \left(\alpha_{2}\right)}{\alpha_{2}-1} x^{-\beta_{2}-1}\left(1+\lambda_{2} x^{-\beta_{2}}\right)^{-\theta_{2}-1} \alpha_{2}^{\left(1+\lambda_{2} x^{-\beta_{2}}\right)^{-\theta_{2}-1}}} .
$$

Then

$$
\begin{array}{r}
\log \frac{f_{1}(x)}{f_{2}(x)}=\log \left\{\frac{\lambda_{1} \beta_{1} \theta_{1} \log \left(\alpha_{1}\right)}{\alpha_{1}-1}\right\}-\left(\beta_{1}+1\right) \log (x)-\left(\theta_{1}+1\right) \log \left(1+\lambda_{1} x^{-\beta_{1}}\right) \\
+\left(1+\lambda_{1} x^{-\beta_{1}}\right)^{-\theta_{1}} \log \left(\alpha_{1}\right)-\log \left\{\frac{\lambda_{2} \beta_{2} \theta_{2} \log \left(\alpha_{2}\right)}{\alpha_{2}-1}\right\}+\left(\beta_{2}+1\right) \log (x) \\
+\left(\theta_{2}+1\right) \log \left(1+\lambda_{2} x^{-\beta_{2}}\right)-\left(1+\lambda_{2} x^{-\beta_{2}}\right)^{-\theta_{2}} \log \left(\alpha_{2}\right) .
\end{array}
$$

If $\alpha_{1}=\alpha_{2}, \lambda_{1}=\lambda_{2}, \theta_{1}=\theta_{2}$ and $\beta_{1} \geq \beta_{2}$, then we have

$$
\begin{aligned}
& \frac{d}{d x} \log \frac{f_{1}(x)}{f_{2}(x)}=-\left(\frac{\beta_{1}+1}{x}\right)+\lambda_{1} \beta_{1}\left(\theta_{1}+1\right)\left(\frac{x^{-\beta_{1}-1}}{1+\lambda_{1} x^{-\beta_{1}}}\right) \\
&+\lambda_{1} \beta_{1} \theta_{1} \log \left(\alpha_{1}\right) x^{-\beta_{1}-1}\left(1+\lambda_{1} x^{-\beta_{1}}\right)^{-\theta_{1}-1}+\left(\frac{\beta_{2}+1}{x}\right) \\
&-\lambda_{2} \beta_{2}\left(\theta_{2}+1\right)\left(\frac{x^{-\beta_{2}-1}}{1+\lambda_{2} x^{-\beta_{2}}}\right) \\
&-\lambda_{2} \beta_{2} \theta_{2} \log \left(\alpha_{2}\right) x^{-\beta_{2}-1}\left(1+\lambda_{2} x^{-\beta_{2}}\right)^{-\theta_{2}-1}<0 .
\end{aligned}
$$

Consequentely, $f_{1}(x) / f_{2}(x)$ decreases in $x$ and hence $X_{1} \leq_{l r} X_{2}$.

\subsection{Stress Strength Model}

Let $X_{1}$ and $X_{2}$ be two independent random variables with $X_{1} \sim \operatorname{APTD}\left(\alpha_{1}, \beta_{1}, \lambda_{1}, \theta_{1}\right)$ and $X_{2} \sim \operatorname{APTD}\left(\alpha_{2}, \beta_{2}, \lambda_{2}, \theta_{2}\right)$ distributions. Then, the stress strength model is given by

$$
R=\operatorname{Pr}\left(X_{2}<X_{1}\right)=\int_{0}^{\infty} f_{1}\left(\alpha_{1}, \beta_{1}, \lambda_{1}, \theta_{1}\right) F_{2}\left(\alpha_{2}, \beta_{2}, \lambda_{2}, \theta_{2}\right) d x
$$




$$
\begin{gathered}
=\underbrace{\Omega \underbrace{\infty}_{0} x^{-\beta_{1}-1}\left(1+\lambda_{1} x^{-\beta_{1}}\right)^{-\theta_{1}-1} e^{\left(1+\lambda_{1} x^{-\beta_{1}}\right)^{-\theta_{1}} \log \left(\alpha_{1}\right)+\left(1+\lambda_{2} x^{-\beta_{2}}\right)^{-\theta_{2}} \log \left(\alpha_{2}\right)}}_{\mathrm{H}} d x \\
-\Omega \underbrace{\Omega \underbrace{\int_{0}^{\infty} x^{-\beta_{1}-1}\left(1+\lambda_{1} x^{-\beta_{1}}\right)^{-\theta_{1}-1} e^{\left(1+\lambda_{1} x^{-\beta_{1}}\right)^{-\theta_{1}} \log \left(\alpha_{1}\right)}}_{0} d x}_{\mathrm{E}}
\end{gathered}
$$

where,

$$
\begin{aligned}
\Omega= & \frac{\lambda_{1} \beta_{1} \theta_{1} \log \left(\alpha_{1}\right)}{\left(\alpha_{1}-1\right)\left(\alpha_{2}-1\right)}, \\
\mathrm{H}= & \sum_{w, h, j=0}^{\infty} \frac{\lambda_{1}^{-\beta_{2} j / \beta_{1}-1} \lambda_{2}^{j}\left(\log \left(\alpha_{1}\right)\right)^{w-h}\left(\log \left(\alpha_{2}\right)\right)^{h}}{\beta_{1} w !} \\
& \times\left(\begin{array}{c}
w \\
h
\end{array}\right)\left(\begin{array}{c}
-\theta_{2} h \\
j
\end{array}\right) B\left(1+\beta_{2} j / \beta_{1}, \theta_{1}(w-h+1)-\beta_{2} j / \beta_{1}\right)
\end{aligned}
$$

and

$$
\mathrm{E}=\sum_{w, \ell=0}^{\infty} \frac{(-1)^{\ell}\left(\log \left(\alpha_{1}\right)\right)^{w}}{w ! \beta_{1} \lambda_{1}\left(\theta_{1}(w+1)+\ell+2\right)}\left(\begin{array}{c}
-2 \\
\ell
\end{array}\right)
$$

Therefore, the stress strength model for the APTD distribution is

$$
R=\sum_{w=0}^{\infty} \eta_{w}\left(\sum_{h, j=0}^{\infty} \delta_{h, j}-\sum_{\ell=0}^{\infty} \tau_{\ell}\right)
$$

where

$$
\begin{aligned}
& \eta_{w}=\frac{\theta_{1}\left(\log \left(\alpha_{1}\right)\right)^{w+1}}{\left(\alpha_{1}-1\right)\left(\alpha_{2}-1\right) w !} \\
& \delta_{h, j}=\lambda_{2}^{j}\left(\log \frac{\alpha_{2}}{\alpha_{1}}\right)^{h} B\left(1+\beta_{2} j / \beta_{1}, \theta_{1}(w-h+1)-\beta_{2} j / \beta_{1}\right),
\end{aligned}
$$

and

$$
\tau_{\ell}=\frac{(-1)^{\ell}}{\theta_{1}(w+1)+\ell+2}\left(\begin{array}{c}
-2 \\
\ell
\end{array}\right) .
$$

\section{Characterization Results}

This section is devoted to the characterizations of the APTD distribution in two directions: (i) based on the ratio of two truncated moments and (ii) in terms of the 
hazard function. Note that (i) can be employed also when the cdf does not have a closed form. We present our characterizations (i)-(ii) in two subsections.

\subsection{Characterizations based on two truncated moments}

This subsection deals with the characterizations of APTD distribution based on the ratio of two truncated moments. Our first characterization employs a theorem due to Glanzel (1987), see Theorem 1 of Appendix A. The result, however, holds also when the interval $H$ is not closed, since the condition of the Theorem is on the interior of $H$.

Proposition 3.1. Let $X: \Omega \rightarrow(0, \infty)$ be a continuous random variable and let, $q_{1}=\alpha^{-\left(1+\lambda x^{-\beta}\right)^{-\theta}}$ and $q_{2}(x)=q_{1}(x)\left(1+\lambda x^{-\beta}\right)^{-\theta}$ for $x>0$. The random variable $X$ has pdf (6) if and only if the function $\xi$ defined in Theorem 1 is of the form

$$
\xi(x)=\frac{1}{2}\left\{1+\left(1+\lambda x^{-\beta}\right)^{-\theta}\right\}, x>0
$$

Proof. Suppose the random variable $X$ has pdf (6), then

$$
(1-F(x)) E\left[q_{1}(X) \mid X \geq x\right]=\frac{\log (\alpha)}{\alpha-1}\left\{1-\left(1+\lambda x^{-\beta}\right)^{-\theta}\right\}, x>0,
$$

and

$$
(1-F(x)) E\left[q_{2}(X) \mid X \geq x\right]=\frac{\log (\alpha)}{2(\alpha-1)}\left\{1-\left(1+\lambda x^{-\beta}\right)^{-2 \theta}\right\}, x>0
$$

Further,

$$
\xi(x) q_{1}(x)-q_{2}(x)=\frac{q_{1}(x)}{2}\left\{1-\left(1+\lambda x^{-\beta}\right)^{-\theta}\right\}>0, \text { for } x>0 .
$$

Conversely, if $\xi$ is of the above form, then

$$
s^{\prime}(x)=\frac{\xi^{\prime}(x) q_{1}(x)}{\xi(x) q_{1}(x)-q_{2}(x)}=\frac{\lambda \beta \theta\left(1+\lambda x^{-\beta}\right)^{-\theta-1}}{1-\left(1+\lambda x^{-\beta}\right)^{-\theta}}, x>0,
$$

and consequently

$$
s(x)=-\log \left\{1-\left(1+\lambda x^{-\beta}\right)^{-\theta}\right\}, x>0 .
$$

Now, according to Theorem 1, $X$ has density (6). 
Corollary 3.1. Let $X: \Omega \rightarrow(0, \infty)$ be a continuous random variable and let $q_{1}(x)$ be as in Proposition 3.1. The random variable $X$ has pdf (6) if and only if there exist functions $q_{2}$ and $\xi$ defined in Theorem 1 satisfying the following differential equation

$$
\frac{\xi^{\prime}(x) q_{1}(x)}{\xi(x) q_{1}(x)-q_{2}(x)}=\frac{\lambda \beta \theta x^{-\beta-1}\left(1+\lambda x^{-\beta}\right)^{-\theta-1}}{1-\left(1+\lambda x^{-\beta}\right)^{-\theta}}, x>0 .
$$

Corollary 3.2. The general solution of the differential equation in Corollary 3.1 is

$$
\xi(x)=\left\{1-\left(1+\lambda x^{-\beta}\right)^{-\theta}\right\}^{-1}\left[-\int \lambda \beta \theta x^{-\beta-1}\left(1+\lambda x^{-\beta}\right)^{-\theta-1}\left(q_{1}(x)\right)^{-1} q_{2}(x) d x+D\right],
$$

where $D$ is a constant. We like to point out that one set of functions satisfying the above differential equation is given in Proposition 3.1 with $D=1 / 2$. Clearly, there are other triplets $\left(q_{1}, q_{2}, \xi\right)$ which satisfy conditions of Theorem 1.

\subsection{Characterization in terms of hazard function}

The hazard function, $\tau_{F}$, of a twice differentiable distribution function, $F$, satisfies the following first order differential equation

$$
\frac{f^{\prime}(x)}{f(x)}=\frac{\tau_{F}^{\prime}(x)}{\tau_{F}(x)}-\tau_{F}(x) \text {. }
$$

It should be mentioned that for many univariate continuous distributions, the above equation is the only differential equation available in terms of the hazard function. In this subsection we present non-trivial characterizations of APTD distribution in terms of the hazard function.

Proposition 3.2. Let $X: \Omega \rightarrow \mathbb{R}$ be a continuous random variable. The random variable $X$ has pdf (6) if and only if its hazard function $\tau_{F}(x)$ satisfies the following differential equation

$$
\tau_{F}^{\prime}(x)-\frac{\lambda \beta \theta \log (\alpha) x^{-\beta-1}}{\left(1+\lambda x^{-\beta}\right)^{\theta+1}} \tau_{F}(x)=\lambda \beta \theta \log (\alpha) \alpha^{-\left(1+\lambda x^{-\beta}\right)^{-\theta}} \frac{d}{d x}\left\{\frac{x^{-\beta-1}\left(1+\lambda x^{-\beta}\right)^{-\theta-1}}{\alpha^{-\left(1+\lambda x^{-\beta}\right)^{-\theta}}-1}\right\}, x>0 .
$$

Proof. If $X$ has pdf (6), then clearly the above differential equation holds. If the differential equation holds, then 


$$
\frac{d}{d x}\left\{\alpha^{-\left(1+\lambda x^{-\beta}\right)^{-\theta}} \tau_{F}(x)\right\}=\lambda \beta \theta \log (\alpha) \frac{d}{d x}\left\{\frac{x^{-\beta-1}\left(1+\lambda x^{-\beta}\right)^{-\theta-1}}{\alpha^{-\left(1+\lambda x^{-\beta}\right)^{-\theta}}-1}\right\}
$$

from which we arrive at the hazard function corresponding to pdf (6).

\section{Maximum Likelihood Estimation}

The maximum likelihood estimates (MLEs) for the model parameters of the APTD distribution will be discussed in this section. Let $x_{1}, x_{2}, \ldots, x_{n}$ be observed values of a random sample from the APTD distribution, then the corresponding log-likelihood function is given by

$$
\begin{aligned}
\ell=n & \{\log (\lambda)+\log (\beta)+\log (\theta)+\log (\log (\alpha))-\log (\alpha-1)\} \\
& -(\beta-1) \sum_{i=1}^{n} \log \left(x_{i}\right)-(\theta-1) \sum_{i=1}^{n} \log \left(1+\lambda x_{i}^{-\beta}\right) \\
& +\log (\alpha) \sum_{i=1}^{n}\left(1+\lambda x_{i}^{-\beta}\right)^{-\theta} .
\end{aligned}
$$

The components of the score vector $\nabla \ell=\left(\frac{\partial \ell}{\partial \alpha}, \frac{\partial \ell}{\partial \beta}, \frac{\partial \ell}{\partial \lambda}, \frac{\partial \ell}{\partial \theta}\right)$ are the following:

$$
\begin{aligned}
& \frac{\partial \ell}{\partial \alpha}=\frac{1}{\alpha}\left\{\frac{n[\alpha(1-\log (\alpha))-1]}{(\alpha-1) \log (\alpha)}\right\}+\sum_{i=1}^{n}\left(1+x_{i}^{-\beta}\right)^{-\theta}, \\
& \frac{\partial \ell}{\partial \beta}=\frac{n}{\beta}-\sum_{i=1}^{n} \log \left(x_{i}\right)+\lambda(\theta+1) \sum_{i=1}^{n}\left(\frac{x_{i}^{-\beta} \log \left(x_{i}\right)}{1+\lambda x_{i}^{-\beta}}\right) \\
& +\lambda(\theta+1) \log (\alpha) \sum_{i=1}^{n}\left(\frac{x_{i}^{-\beta} \log \left(x_{i}\right)}{\left(1+\lambda x_{i}^{-\beta}\right)^{\theta+1}}\right), \\
& \frac{\partial \ell}{\partial \lambda}=\frac{n}{\lambda}-(\theta+1) \sum_{i=1}^{n}\left(\frac{x_{i}^{-\beta}}{1+\lambda x_{i}^{-\beta}}\right)-\theta \log (\alpha) \sum_{i=1}^{n}\left(\frac{x_{i}^{-\beta}}{\left(1+\lambda x_{i}^{-\beta}\right)^{\theta+1}}\right),
\end{aligned}
$$

and

$$
\frac{\partial \ell}{\partial \theta}=\frac{n}{\theta}-\sum_{i=1}^{n} \log \left(1+\lambda x_{i}^{-\beta}\right)+\log (\alpha) \sum_{i=1}^{n}\left(\frac{\log \left(1+\lambda x_{i}^{-\beta}\right)}{\left(1+\lambda x_{i}^{-\beta}\right)^{\theta}}\right) .
$$


The MLEs, say $\hat{\Theta}=(\hat{\alpha}, \hat{\beta}, \hat{\lambda}, \hat{\theta})$, of $\Theta=(\alpha, \beta, \lambda, \theta)^{T}$ can be obtained by equating the system of nonlinear equations (23) to (26) to zero and solving them simultaneously. The components of the observed information matrix of the model parameters are obtained in Appendix B.

\section{Simulation Study}

In this section, we evaluate the performance of the MLEs of the model parameters of the APTD distribution via a Monte Carlo simulation. We compare MLEs to minimum spacing absolute distance estimator (MSADE) and minimum spacing absolute-log distance estimator (MSALDE) of the APTD distribution (see Torabi and Bagheri (2010) and Torabi and Montazeri (2014)). The simulation is performed for sample size $n=50,100,200,300$ and 500. The parameters values are $\alpha=1.5, \beta=1.0, \theta=0.5$ and $\lambda=0.8$. For each sample size, we compute the MLEs, MSADEs and MSALDEs of the parameters. We repeat this process 3,000 times and obtain the average estimates (AEs), biases and mean square error (MSEs). The results are reported in Table 1. It is observed that the MSEs of MLE is less than MSEs of MSADEs and MSALDEs for large sample size. We can verify that the estimates are stable and quite close to the true parameter values for these sample sizes. As the sample size increases the MSE decreases in all cases. Accordingly, the sample size $n$ plays an important role in determining the efficiency of the parameters since when $n$ increases some additional information is gathered. 
Table 1: Estimated AE and MSE of MLE, MSADE and MSALDE of the model parameters of the APTD distribution.

\begin{tabular}{|c|c|c|c|c|c|c|c|c|c|c|}
\hline \multicolumn{2}{|c|}{ Different method } & \multicolumn{3}{|c|}{ MLE } & \multicolumn{3}{|c|}{ MSADE } & \multicolumn{3}{|c|}{ MSALDE } \\
\hline$n$ & Parameter & A.E & Bias & MSE & A.E & Bias & MSE & A.E & Bias & MSE \\
\hline \multirow{4}{*}{50} & $\alpha$ & 2.022 & 0.522 & 2.948 & 1.614 & 0.114 & 1.004 & 0.574 & 0.074 & 0.067 \\
\hline & $\beta$ & 2.346 & 1.346 & 6.872 & 1.325 & 0.325 & 2.526 & 1.335 & 0.335 & 3.003 \\
\hline & $\theta$ & 0.551 & 0.081 & 0.092 & 0.517 & 0.117 & 0.144 & 0.600 & 0.100 & 0.099 \\
\hline & $\lambda$ & 0.975 & 0.175 & 0.248 & 0.863 & 0.063 & 0.093 & 0.835 & 0.035 & 0.169 \\
\hline \multirow{4}{*}{100} & $\alpha$ & 1.693 & 0.193 & 0.689 & 1.597 & 0.097 & 0.641 & 1.507 & 0.007 & 0.727 \\
\hline & $\beta$ & 1.328 & 0.328 & 3.630 & 1.210 & 0.210 & 2.079 & 1.325 & 0.325 & 2.878 \\
\hline & $\theta$ & 0.553 & 0.053 & 0.087 & 0.531 & 0.091 & 0.103 & 0.598 & 0.098 & 0.087 \\
\hline & $\lambda$ & 0.915 & 0.115 & 0.197 & 0.824 & 0.024 & 0.092 & 0.798 & -0.002 & 0.163 \\
\hline \multirow{4}{*}{200} & $\alpha$ & 1.549 & 0.049 & 0.348 & 1.492 & -0.008 & 0.326 & 1.401 & -0.099 & 0.392 \\
\hline & $\beta$ & 1.164 & 0.164 & 0.909 & 1.147 & 0.147 & 0.505 & 1.226 & 0.226 & 0.938 \\
\hline & $\theta$ & 0.560 & 0.051 & 0.084 & 0.549 & 0.081 & 0.097 & 0.603 & 0.083 & 0.083 \\
\hline & $\lambda$ & 0.873 & 0.073 & 0.162 & 0.800 & -0.001 & 0.086 & 0.776 & -0.024 & 0.136 \\
\hline \multirow{4}{*}{300} & $\alpha$ & 1.543 & 0.043 & 0.232 & 1.482 & -0.018 & 0.252 & 1.420 & -0.080 & 0.300 \\
\hline & $\beta$ & 1.084 & 0.084 & 0.584 & 1.091 & 0.091 & 0.377 & 1.147 & 0.147 & 0.667 \\
\hline & $\theta$ & 0.530 & 0.030 & 0.031 & 0.540 & 0.038 & 0.049 & 0.579 & 0.079 & 0.066 \\
\hline & $\lambda$ & 0.870 & 0.070 & 0.123 & 0.802 & 0.002 & 0.074 & 0.794 & -0.006 & 0.117 \\
\hline \multirow{4}{*}{500} & $\alpha$ & 1.520 & 0.020 & 0.033 & 1.451 & -0.197 & 0.049 & 1.419 & -0.081 & 0.241 \\
\hline & $\beta$ & 1.000 & -0.001 & 0.306 & 1.077 & 0.077 & 0.311 & 1.081 & 0.081 & 0.417 \\
\hline & $\theta$ & 0.519 & 0.019 & 0.027 & 0.541 & 0.031 & 0.035 & 0.565 & 0.065 & 0.055 \\
\hline & $\lambda$ & 0.866 & 0.066 & 0.050 & 0.796 & -0.004 & 0.069 & 0.800 & 0.001 & 0.103 \\
\hline
\end{tabular}

\section{Applications}

In this section, we introduce three applications to real data sets to illustrate the usefulness of the APTD distribution. The first data set consists of 63 observations of the strengths of $1.5 \mathrm{~cm}$ glass fibers which obtained by workers at the UK National Physical Laboratory. The data are: $0.55,0.74,0.77,0.81,0.84,0.93,1.04,1.11,1.13,1.24,1.25$, $1.27,1.28,1.29,1.30,1.36,1.39,1.42,1.48,1.48,1.49,1.49,1.50,1.50,1.51,1.52,1.53$, $1.54,1.55,1.55,1.58,1.59,1.60,1.61,1.61,1.61,1.61,1.62,1.62,1.63,1.64,1.66,1.66$, $1.66,1.67,1.68,1.68,1.69,1.70,1.70,1.73,1.76,1.76,1.77,1.78,1.81,1.82,1.84,1.84$, 
$1.89,2.00,2.01,2.24$. This data have also been used by Smith and Naylor (1987) and Merovci et al. (2016).

The second data set (Cooray and Ananda, 2008) represents the failure times of Kevlar 49/epoxy strands when the pressure is at 90\% stress level: 0.01, 0.01, 0.02, 0.02, $0.02,0.03,0.03,0.04,0.05,0.06,0.07,0.07,0.08,0.09,0.09,0.10,0.10,0.11,0.11,0.12$, $0.13,0.18,0.19,0.20,0.23,0.24,0.24,0.29,0.34,0.35,0.36,0.38,0.40,0.42,0.43,0.52$, $0.54,0.56,0.60,0.60,0.63,0.65,0.67,0.68,0.72,0.72,0.72,0.73,0.79,0.79,0.80,0.80$, 0.83, 0.85, 0.90, 0.92, 0.95, 0.99, 1.00, 1.01, 1.02, 1.03, 1.05, 1.10, 1.10, 1.11, 1.15, 1.18, $1.20,1.29,1.31,1.33,1.34,1.40,1.43,1.45,1.50,1.51,1.52,1.53,1.54,1.54,1.55,1.58$, $1.60,1.63,1.64,1.80,1.80,1.81,2.02,2.05,2.14,2.17,2.33,3.03,3.03,3.34,4.20,4.69$, 7.89 .

The second data set represents the survival times (in days) of 72 guinea pigs infected with virulent tubercle bacilli (Bjerkedal, 1960). Guinea pigs are known to have high susceptibility of human tuberculosis, which is one of the reasons for choosing this species. The survival times of the Guinea pigs in days are: 0.1,0.33,0.44,0.56,0.59, $0.72,0.74,0.77,0.92,0.93,0.96,1,1,1.02,1.05,1.07,07, .08,1.08,1.08,1.09,1.12,1.13$, $1.15,1.16,1.2,1.21,1.22,1.22,1.24,1.3,1.341 .36,1.39,1.44,1.46,1.53,1.59,1.6,1.63$, $1.63,1.68,1.71,1.72,1.76,1.83,1.95,1.96,1.97,2.02,2.13,2.15,2.16,2.22,2.3,2.31$, $2.4,2.45,2.51,2.53,2.54,2.54,2.78,2.93,3.27,3.42,3.47,3.61,4.02,4.32,4.58,5.55$.

We estimate the unknown parameters of all competitive models by maximum likelihood. We compute the log-likelihood function evaluated at the MLEs using a limited memory quasi-Newton code for bound-constrained optimization (L-BFGS-B). For model comparison, we consider six well-known statistics: the maximized loglikelihood (LL), Akaike information criterion (AIC), BIC (Bayesian information criterion), Anderson-Darling $\left(A^{*}\right)$, Cram'er von Mises, $\left(W^{*}\right)$ and Kolmogorov-Smirnov (K-S) measures, where lower values of these statistics and higher p-values of $K-S$ indicate good fits.

We compare the APTD distribution with those of alpha power Weibull (APW) (Nassar et al., 2016), exponentiated Kumaraswamy Dagum (EKD) (Huang and Oluyede; 
2014), Beta Dagum (BD)(Domma and Condino; 2013) and Weibull Dagum (WD) (Tahir et al., 2016).

The densities of the competitive models are, respectively, given by

$$
\begin{aligned}
f_{A P W}= & \frac{\lambda \beta \log (\alpha)}{\alpha-1} x^{\beta-1} e^{-\lambda x^{\beta}} \alpha^{1-e^{-\lambda x^{\beta}}}, x, \alpha, \beta, \lambda>0, \alpha \neq 1, \\
f_{E K D}= & \alpha \lambda \delta \phi \theta x^{-\delta-1}\left(1+\lambda x^{-\delta}\right)^{-\alpha-1}\left[1-\left(1+\lambda x^{-\delta}\right)^{-\alpha}\right]^{\phi-1} \\
& \times\left\{1-\left[1-\left(1+\lambda x^{-\delta}\right)^{-\alpha}\right]^{\phi}\right\}^{\theta-1}, x, \alpha, \lambda, \delta, \phi, \theta>0, \\
f_{B D}= & \frac{\beta \lambda \delta}{B(a, b)} x^{-\delta-1}\left(1+\lambda x^{-\delta}\right)^{-a \beta-1}\left[1-\left(1+\lambda x^{-\delta}\right)^{-\beta}\right]^{b-1}, x, \lambda, \beta, \delta, a, b>0,
\end{aligned}
$$

and

$$
\begin{aligned}
f_{W D}=b \lambda & \delta \beta x^{-\delta-1}\left(1+\lambda x^{-\delta}\right)^{-b \beta-1}\left[1-\left(1+\lambda x^{-\delta}\right)^{-\beta}\right]^{-b-1} \\
\times & e^{\left[\left(1+\lambda x^{-\delta}\right)^{\beta}-1\right]^{-b}}, x, \lambda, \beta, \delta, b>0 .
\end{aligned}
$$

\begin{tabular}{|c|c|c|c|c|c|c|c|c|}
\hline \multirow{2}{*}{ Distribution } & \multicolumn{7}{|c|}{ Estimates with standard error in parenthesis } & \multirow[b]{2}{*}{$\hat{b}$} \\
\hline & $\hat{\alpha}$ & $\hat{\beta}$ & $\hat{\lambda}$ & $\hat{\theta}$ & $\hat{\delta}$ & $\hat{\phi}$ & $\hat{a}$ & \\
\hline \multirow{2}{*}{ APTD } & 16.345 & 12.479 & 217.251 & 0.246 & \multirow{2}{*}{--- } & \multirow{2}{*}{---} & \multirow{2}{*}{--- } & \multirow{2}{*}{---} \\
\hline & (3.195) & $(1.311)$ & $(11.724)$ & $(0.108)$ & & & & \\
\hline \multirow{2}{*}{ APW } & 10.843 & 4.483 & 0.195 & 8.792 & 3.196 & 193.570 & & \\
\hline & $(1.658)$ & $(0.761)$ & $(0.108)$ & $(0.011)$ & $(0.011)$ & $(0.011)$ & & \\
\hline \multirow{2}{*}{ EKD } & 0.177 & \multirow[b]{2}{*}{---} & 5.068 & \multirow[b]{2}{*}{---} & \multirow[b]{2}{*}{---} & \multirow[b]{2}{*}{---} & \multirow[b]{2}{*}{---} & \multirow[b]{2}{*}{---} \\
\hline & $(0.022)$ & & $(0.033)$ & & & & & \\
\hline \multirow{2}{*}{ BD } & \multirow{2}{*}{---} & 72.416 & 2.198 & \multirow{2}{*}{---} & 0.455 & \multirow{2}{*}{---} & 4.826 & 2.652 \\
\hline & & (10.247) & (2.183) & & $(0.256)$ & & (1.822) & (1.383) \\
\hline \multirow{2}{*}{ WD } & \multirow{2}{*}{--- } & 14.938 & 52.659 & \multirow{2}{*}{--- } & 0.158 & \multirow{2}{*}{--- } & \multirow{2}{*}{--- } & 1.262 \\
\hline & & (3.267) & (34.284) & & $(0.028)$ & & & $(0.360)$ \\
\hline
\end{tabular}

The MLEs and some statistics of the models for the data sets are introduced in Tables (2), (3), (4), (5), (6) and (7) respectively. The estimated pdfs and cdfs plots of all competitive distributions for the three data sets are displayed in Figures 4, 5 and 6 respectively.

Table 2: The MLEs for the first data set. 
Table 3: Some statistics for the models fitted to the first data set.

\begin{tabular}{cccccccc}
\hline \multirow{2}{*}{ Distribution } & \multicolumn{7}{c}{ Goodness-0f-fit statistics } \\
\cline { 2 - 8 } & $\mathrm{LL}$ & $\mathrm{AIC}$ & $\mathrm{BIC}$ & $\mathrm{A}^{*}$ & $\mathrm{~W}^{*}$ & $\mathrm{~K}-\mathrm{S}$ & P-value \\
\hline APTD & 11.662 & 31.324 & 39.190 & 0.817 & 0.149 & 0.117 & 0.356 \\
\hline APW & 13.474 & 32.948 & 39.378 & 0.928 & 0.169 & 0.123 & 0.300 \\
\hline EKD & 13.823 & 37.646 & 48.362 & 0.997 & 0.181 & 0.151 & 0.113 \\
\hline BD & 14.283 & 38.566 & 49.282 & 1.038 & 0.187 & 0.144 & 0.146 \\
\hline WD & 14.736 & 37.471 & 46.044 & 1.164 & 0.211 & 0.147 & 0.134 \\
\hline
\end{tabular}
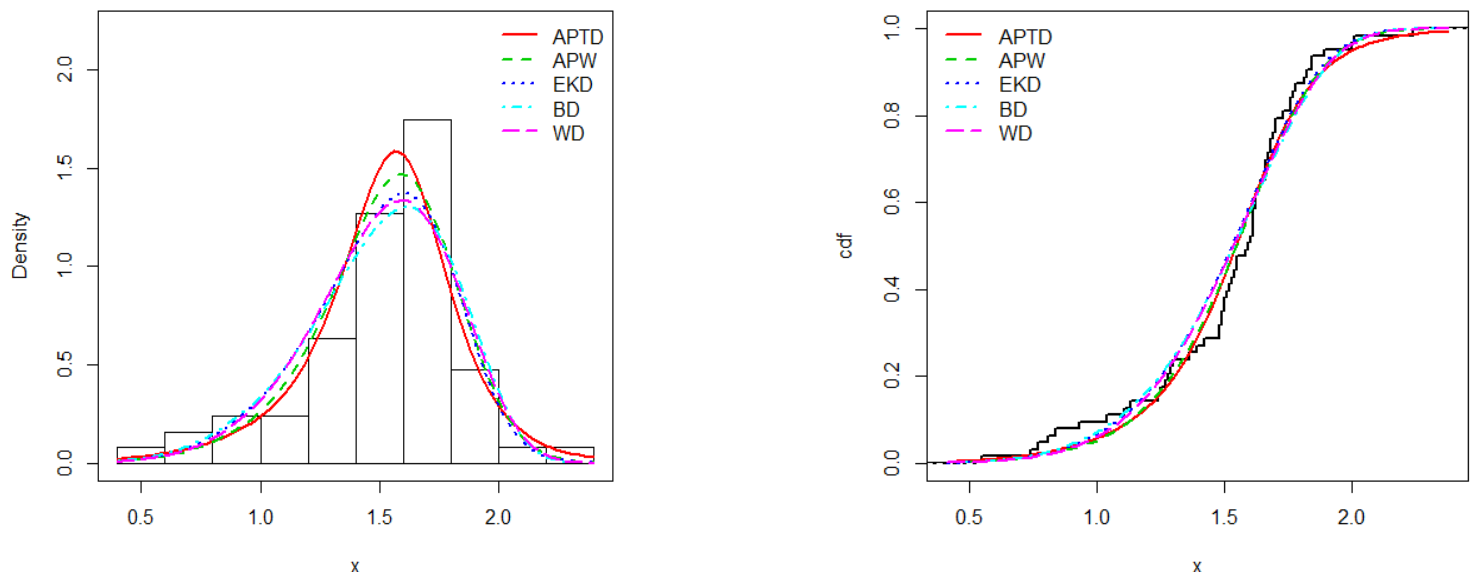

Figure 4: Estimated pdfs and cdfs plots of the APTD distribution for data set 1.

Table 4: The MLEs for the second data set.

\begin{tabular}{|c|c|c|c|c|c|c|c|c|}
\hline \multirow{2}{*}{ Distribution } & \multicolumn{7}{|c|}{ Estimates with standard error in parenthesis } & \multirow[b]{2}{*}{$\hat{b}$} \\
\hline & $\hat{\alpha}$ & $\hat{\beta}$ & $\hat{\lambda}$ & $\hat{\theta}$ & $\hat{\delta}$ & $\hat{\phi}$ & $\hat{a}$ & \\
\hline \multirow{2}{*}{ APTD } & 1.452 & 3.370 & 7.277 & 0.202 & \multirow{2}{*}{---} & \multirow{2}{*}{---} & \multirow{2}{*}{---} & \multirow{2}{*}{---} \\
\hline & $(1.521)$ & $(0.687)$ & $(2.778)$ & $(0.060)$ & & & & \\
\hline \multirow{2}{*}{ APW } & 3.559 & 0.806 & 1.404 & \multirow{2}{*}{---} & \multirow{2}{*}{---} & \multirow{2}{*}{---} & \multirow{2}{*}{---} & \multirow{2}{*}{---} \\
\hline & (3.937) & $(0.128)$ & $(0.373)$ & & & & & \\
\hline \multirow{2}{*}{ EKD } & 0.343 & \multirow{2}{*}{---} & 14.762 & 0.975 & 2.462 & 3.933 & & \\
\hline & $(0.715)$ & & $(5.768)$ & $(0.138)$ & $(2.308)$ & $(0.042)$ & & \\
\hline \multirow{2}{*}{$\mathrm{BD}$} & \multirow{2}{*}{---} & 54.532 & 0.821 & \multirow{2}{*}{---} & 0.806 & \multirow{2}{*}{---} & 1.281 & 58.215 \\
\hline & & (12.323) & $(0.014)$ & & $(0.423)$ & & $(0.945)$ & (4.171) \\
\hline \multirow{2}{*}{ WD } & \multirow{2}{*}{--- } & 5.999 & 55.406 & \multirow{2}{*}{--- } & 0.415 & \multirow{2}{*}{--- } & \multirow{2}{*}{---} & 0.232 \\
\hline & & (6.111) & (5.427) & & $(0.474)$ & & & $(0.246)$ \\
\hline
\end{tabular}


Table 5: Some statistics for the models fitted to the second data set.

\begin{tabular}{cccccccc}
\hline \multirow{2}{*}{ Distribution } & \multicolumn{7}{c}{ Goodness-of-fit statistics } \\
\cline { 2 - 8 } & $\mathrm{LL}$ & AIC & BIC & $\mathrm{A}^{*}$ & $\mathrm{~W}^{*}$ & $\mathrm{~K}-\mathrm{S}$ & P-value \\
\hline APTD & 100.044 & 208.089 & 218.549 & 0.464 & 0.066 & 0.065 & 0.782 \\
\hline APW & 102.369 & 210.738 & 218.583 & 0.923 & 0.159 & 0.076 & 0.602 \\
\hline EKD & 102.253 & 214.505 & 227.581 & 0.839 & 0.141 & 0.077 & 0.594 \\
\hline BD & 102.754 & 215.508 & 228.584 & 0.986 & 0.172 & 0.084 & 0.475 \\
\hline WD & 102.883 & 213.765 & 224.226 & 0.969 & 0.167 & 0.084 & 0.475 \\
\hline
\end{tabular}
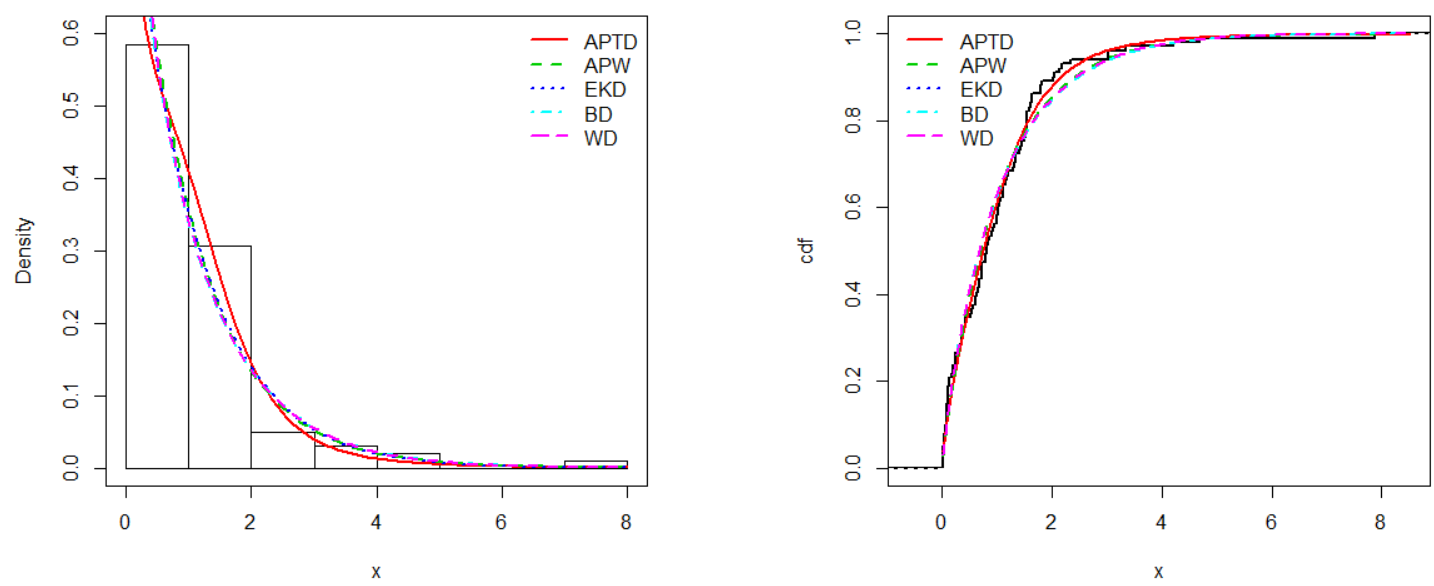

Figure 5: Estimated pdfs and cdfs plots of the APTD distribution for data set 2.

Table 6: The MLEs for the third data set.

\begin{tabular}{ccccccccc}
\hline \multirow{2}{*}{ Distribution } & \multicolumn{7}{c}{ Estimates with standard error in parenthesis } \\
\cline { 2 - 9 } & $\hat{\alpha}$ & $\hat{\beta}$ & $\hat{\lambda}$ & $\hat{\theta}$ & $\hat{\delta}$ & $\hat{\phi}$ & $\hat{a}$ & $\hat{b}$ \\
\hline \multirow{2}{*}{ APTD } & 51.948 & 3.065 & 2.980 & 0.331 & -- & --- & --- & --- \\
& $(11.254)$ & $(0.476)$ & $(2.369)$ & $(0.224)$ & & & & \\
\hline \multirow{2}{*}{ APW } & 243.092 & 0.898 & 1.357 & & & & & \\
& $(94.320)$ & $(0.135)$ & $(0.289)$ & -- & --- & --- & -- & -- \\
\hline \multirow{2}{*}{ EKD } & 4.175 & & 0.239 & 9.295 & 0.009 & 1.774 & & - \\
& $(2.085$ & --- & $(0.331)$ & $(1.202)$ & $(0.027)$ & $(4.241)$ & & -- \\
\hline \multirow{2}{*}{ BD } & --- & 1.014 & 1.187 & & 0.484 & & 3.335 & 2.035 \\
& & $(0.814)$ & $(1.249)$ & -- & $(0.476)$ & & $(1.580)$ & $(0.489)$ \\
\hline \multirow{2}{*}{ WD } & --- & 12.128 & 20.565 & & 0.794 & & & 0.144 \\
& & $(4.430)$ & $(8.774)$ & -- & $(0.388)$ & & - & $(0.191)$ \\
\hline
\end{tabular}


Table 7: Some statistics for the models fitted to the third data set.

\begin{tabular}{cccccccc}
\hline \multirow{2}{*}{ Distribution } & \multicolumn{7}{c}{ Goodness-of-fit statistics } \\
\cline { 2 - 8 } & $\mathrm{LL}$ & AIC & BIC & $\mathrm{A}^{*}$ & $\mathrm{~W}^{*}$ & $\mathrm{~K}-\mathrm{S}$ & P-value \\
\hline APTD & 99.223 & 207.847 & 216.953 & 0.252 & 0.040 & 0.065 & 0.920 \\
\hline APW & 101.156 & 208.312 & 215.142 & 0.489 & 0.078 & 0.082 & 0.719 \\
\hline EKD & 99.987 & 209.174 & 220.558 & 0.278 & 0.048 & 0.069 & 0.882 \\
\hline BD & 101.231 & 212.462 & 223.846 & 0.407 & 0.059 & 0.0870 & 0.648 \\
\hline WD & 104.081 & 216.161 & 225.268 & 0.985 & 0.162 & 0.114 & 0.303 \\
\hline
\end{tabular}
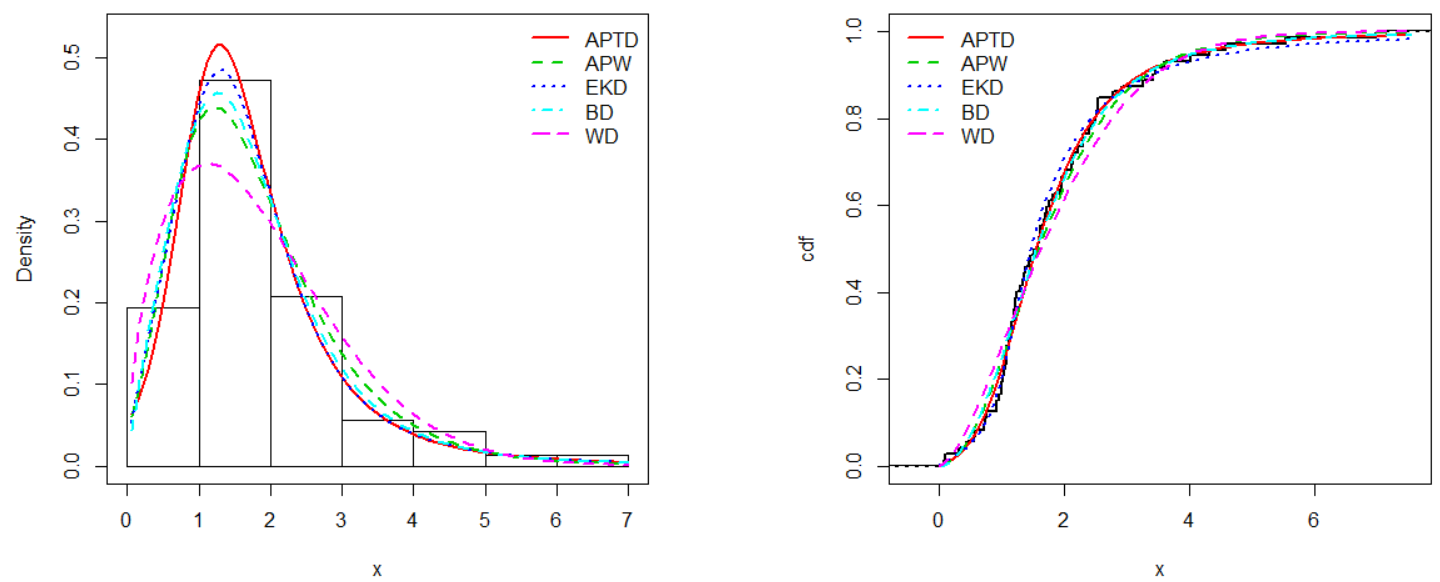

Figure 6: Estimated pdfs and cdfs plots of the APTD distribution for data set 3.

The values in Tables 3, 5 and 7 showed that the APTD distribution has the smallest values for $A^{*}, W^{*}, A I C, B I C, K S$ and largest $p$-values among all competetive models then, it could be chosen as the best model. It is clear from Figures 4, 5 and 6 that the new APTD distribution provides the best fits for the three data sets.

\section{Conclusions}

We introduce a new four parameter Dagum distribution called the alpha power transformed Dagum distribution distribution. The main features of the new model such as the quantile function, ordinary and incomplete moments, moment generating function, order statistics, Rényi entropy, stress strength model and stochastic ordering are investigated. The characterizations of the proposed model are studied. The maximum likelihood criterion is used to estimate the model parameters and the importance of 
these estimates are assesed by means of a simulation study. The usefulness of the new model is illustrated via three real applications. Numerical results show that the new distribution can be considered a good alternative model to the alpha power Weibull, exponentiated Kumaraswamy Dagum, Beta Dagum and Weibull Dagum Distributions.

\section{REFERENCES}

[1] H. Bakouch, M. Khan and C. Chesneau, A power log-Dagum distribution: Estimation with applications to hydrology data; <hal-01491483v1>, (2017).

[2] T. Bjerkedal, Acquisition of resistance in guinea pigs infected with different doses of virulent tubercle bacilli. Amer. J. Hygiene, 72 (1960) 130-148.

[3] K. Cooray and M. Ananda, A generalization of the half-normal distribution with applications to lifetime data. Commun. Stat.-Theory Meth. 37 (2008) 1323-1337.

[4] C. Dagum, New model of personal income-distribution-specification and estimation. Econ. Appl. 30(3) (1977) 413-437.

[5] S. Dey, A. Alzaatreh, C. Zhang and D. Kumar, A new extension of generalized exponential distribution with application to Ozone data, Ozone: Science \& Engineering, J. Int. Ozone Assoc. 39(4) (2017) 273-285.

[6] S. Dey, V. K. Sharma and M. Mesfioui, A new extension of Weibull distribution with application to lifetime data, Ann. Data. Sci. 4 (2017) 31-61.

[7] F. Domma and F. Condino, The beta-dagum distribution: definition and properties, Commun. Stat.-Theory Meth. 42(22) (2013) 4070-4090.

[8] F. Domma, A. Eftekharian, A. Z. Afify, M. Alizadeh and I. Ghosh, The odd log-Logistic Dagum distribution:properties and applications. Rev. Colomb. Estadistica 4(1) (2018), 109-135.

[9] W. Clanzel, A characterization theorem based on truncated moments and its application to some distribution families. Mathematical Statistics and Probability Theory (Bad Tatzmannsdorf, 1986) 7, Reidel, Dordrecht: 75-84. (1987).

[10] W. Glanzel, Some consequences of a characterization theorem based on truncated moments. Statistics 21(4) (1990) 613-618.

[11] S. Huang, B. O. Oluyede, Exponentiated Kumaraswamy-Dagum distribution with applications to income and lifetime data, J. Stat. Distrib. Appl. 1 (2014) 8.

[12] A. Mahdavi and D. Kundu, A new method for generating distributions with an application to Exponential distribution. Commun. Stat.-Theory Meth. 46(13) (2017) 6543-6557. 
[13] F. Merovci, M. A. Khaleel, N. A. Ibrahim and M. Shitan, The beta Burr type $x$ distribution properties with applications, Springer Plus 5 (2016) 697.

[14] M. Nassar, A. Alzaatreh, M. Mead, O. Abo-Kasem, Alpha power Weibull distribution: Properties and applications, Commun. Stat. - Theory Meth. 46 (2017) 10236-10252.

[15] B. O. Oluyede and Y. Ye, Weighted Dagum and related distributions. Afrika Mat. 25(4) (2014) $1125-1141$.

[16] M. Shaked and J. G. Shanthikumar, Stochastic Orders. Wiley: New York. (2007).

[17] R. L. Smith and J. C. Naylor, A comparison of maximum likelihood estimators for the threeparameter Weibull distribution, Appl. Stat. 36 (1987) 358-369.

[18] M. H. Tahir, G. M. Cordeiro, M. Mansoor, M. Zubair and M. Alizadeh, The Weibull-Dagum distribution:properties and applications. Commun. Stat.-Theory Meth. 45(24) (2016) 7376-7398.

[19] H. Torabi and F. L. Bagheri, Estimation of parameters for an extended generalized half logistic distribution based on complete and densored data, J. Iran. Stat. Soc. 9(2) (2010) 171-195.

[20] H. Torabi and N. H. Montazari, The logistic-uniform distribution and its application, Commun. Stat.-Simul. Comput. 43 (2014) 2551-2569. 


\section{Appendix A}

Theorem 1. Let $(\Omega, F, \mathrm{P})$ be a given probability space and let $H=[a, b]$ be an interval for some $d<b \quad(a=-\infty, b=\infty$ might as well be allowed). Let $X: \Omega \rightarrow H$ be a continuous random variable with the distribution function $F$ and let $q_{1}$ and $q_{2}$ be two real functions defined on $H$ such that

$$
E\left[q_{2}(X) \mid X \geq x\right]=E\left[q_{1}(X) \mid X \geq x\right] \xi(x), x \in H,
$$

is defined with some real function $\eta$. Assume that $q_{1}, q_{2} \in C^{-1}(H), \xi \in C^{2}(H)$ and $F$ is twice continuously differentiable and strictly monotone function on the set $H$. Finally, assume that the equation $\xi q_{1}=q_{2}$ has no real solution in the interior of $H$. Then $F$ is uniquely determined by the functions $q_{1}, q_{2}$ and $\xi$, particularly

$$
F(x)=\int_{a}^{x} C\left|\frac{\xi^{\prime}(u)}{\xi(u) q_{1}(u)-q_{2}(u)}\right| \exp (-s(u)) d u,
$$

where the function $s$ is a solution of the differential equation $s^{\prime}=\frac{\xi^{\prime} q_{1}}{\xi q_{1}-q_{2}}$ and $C$ is the normalization constant, such that $\int_{H} d F=1$.

We like to mention that this kind of characterization based on the ratio of truncated moments is stable in the sense of weak convergence (see, Glanzel (1990)), in particular, let us assume that there is a sequence $\left\{X_{n}\right\}$ of random variables with distribution function $\left\{F_{n}\right\}$ such that the functions $q_{1 n}, q_{2 n}$ and $\xi_{n}(n \in N)$ satisfy the conditions of Theorem 1 and let $q_{1 n} \rightarrow q_{1}, q_{2 n} \rightarrow q_{2}$ for some continuously differentiable real functions $q_{1}$ and $q_{2}$. Let, finally, $X$ be a random variable with distribution $F$. Under the condition that $q_{1 n}(X)$ and $q_{2 n}(X)$ are uniformly integrable and the family $\left\{F_{n}\right\}$ is relatively compact, the sequence $X_{n}$ converges to $X$ in distribution if and only if $\xi_{n}$ converges to $\xi$, where

$$
\xi(x)=\frac{E\left[q_{2}(X) \mid X \geq x\right]}{E\left[q_{1}(X) \mid X \geq x\right]} .
$$

This stability theorem makes sure that the convergence of distribution function is reflected by corresponding convergence of the function $q_{1}, q_{2}$ and $\xi_{n}$, respectively. It guarantees, for instance, the convergence of characterization on the Wald distribution to that of the Levy-Smirrnov distribution if $\alpha \rightarrow \infty$.

A further consequence of the stability property of Theorem 1 is the application of this theorem to special tasks in statistical practice such as the estimation of the parameters of discrete distributions. For such purpose, the functions $q_{1}, q_{2}$ and, specially, $\xi$ should be as simple as possible. Since the function triplet is not uniquely determined it is often possible to choose $\xi$ as a linear function. Therefore, it is worth analyzing some special cases which helps to find new characterizations reflecting the relationship between individual continuous univariate distributions and appropriate in other areas of statistics. 


\section{Appendix B}

The components of the observed information matrix are the following

$$
\frac{\partial^{2} \ell}{\partial \alpha^{2}}=n\left(\frac{1}{(\alpha-1)^{2}}-\frac{(1+\log (\alpha))}{(\alpha \log (\alpha))^{2}}\right)-\frac{1}{\alpha^{2}} \sum_{i=1}^{n}\left(1+x_{i}^{-\beta}\right)^{-\theta} \text {, }
$$$$
\frac{\partial^{2} \ell}{\partial \beta \partial \alpha}=\frac{\lambda \theta}{\alpha} \sum_{i=1}^{n}\left(\frac{x_{i}^{-\beta} \log \left(x_{i}\right)}{\left(1+\lambda x_{i}^{-\beta}\right)^{\theta+1}}\right)
$$$$
\frac{\partial^{2} \ell}{\partial \lambda \partial \alpha}=\frac{-\theta}{\alpha} \sum_{i=1}^{n}\left(\frac{x_{i}^{-\beta}}{\left(1+\lambda x_{i}^{-\beta}\right)^{\theta+1}}\right)
$$

$$
\frac{\partial^{2} \ell}{\partial \theta \partial \alpha}=-\left(\frac{1}{\alpha}\right) \sum_{i=1}^{n}\left(\frac{\log \left(1+\lambda x_{i}^{-\beta}\right)}{\left(1+\lambda x_{i}^{-\beta}\right)^{\theta}}\right)
$$

$$
\frac{\partial^{2} \ell}{\partial \beta^{2}}=\frac{-n}{\beta^{2}}-\lambda(\theta+1) \sum_{i=1}^{n}\left(\frac{x_{i}^{-\beta}\left(\log \left(x_{i}\right)\right)^{2}}{\left(1+\lambda x_{i}^{-\beta}\right)^{2}}\right)+\lambda \theta \log (\alpha) \sum_{i=1}^{n}\left(\frac{x_{i}^{-\beta}\left(\log \left(x_{i}\right)\right)^{2}\left(\theta \lambda x_{i}^{-\beta}-1\right)}{\left(1+\lambda x_{i}^{-\beta}\right)^{\theta+2}}\right) \text {, }
$$$$
\frac{\partial^{2} \ell}{\partial \lambda \partial \beta}=(\theta+1) \sum_{i=1}^{n}\left(\frac{x_{i}^{-\beta} \log \left(x_{i}\right)}{\left(1+\lambda x_{i}^{-\beta}\right)^{2}}\right)+\theta \log (\alpha) \sum_{i=1}^{n}\left(\frac{x_{i}^{-\beta} \log \left(x_{i}\right)\left(1-\theta \lambda x_{i}^{-\beta}\right)}{\left(1+\lambda x_{i}^{-\beta}\right)^{2 \theta+1}}\right),
$$$$
\frac{\partial^{2} \ell}{\partial \theta \partial \beta}=\lambda\left\{\sum_{i=1}^{n}\left(\frac{x_{i}^{-\beta} \log \left(x_{i}\right)}{1+\lambda x_{i}^{-\beta}}\right)+\log (\alpha) \sum_{i=1}^{n}\left(\frac{x_{i}^{-\beta} \log \left(x_{i}\right)\left(1-\log \left(1+\lambda x_{i}^{-\beta}\right)\right)}{\left(1+\lambda x_{i}^{-\beta}\right)^{2 \theta}}\right)\right\}
$$$$
\frac{\partial^{2} \ell}{\partial \lambda^{2}}=\frac{-n}{\lambda^{2}}+(\theta+1)\left\{\sum_{i=1}^{n}\left(\frac{x_{i}^{-2 \beta}}{\left(1+\lambda x_{i}^{-\beta}\right)^{2}}\right)+\log (\alpha) \sum_{i=1}^{n}\left(\frac{x_{i}^{-2 \beta}}{\left(1+\lambda x_{i}^{-\beta}\right)^{\theta+2}}\right)\right\}
$$$$
\frac{\partial^{2} \ell}{\partial \theta \partial \lambda}=-\sum_{i=1}^{n}\left(\frac{x_{i}^{-\beta}}{1+\lambda x_{i}^{-\beta}}\right)-\log (\alpha) \sum_{i=1}^{n}\left(\frac{x_{i}^{-\beta}\left(1-\theta \log \left(1-\theta \lambda x_{i}^{-\beta}\right)\right)}{\left(1+\lambda x_{i}^{-\beta}\right)^{\theta+1}}\right)
$$

and

$$
\frac{\partial^{2} \ell}{\partial \theta^{2}}=\frac{-n}{\theta^{2}}+\log (\alpha) \sum_{i=1}^{n}\left(\frac{\left(\log \left(1+\lambda x_{i}^{-\beta}\right)\right)^{2}}{\left(1+\lambda x_{i}^{-\beta}\right)^{\theta}}\right)
$$

